\title{
LA DINÁMICA Y LOS DETERMINANTES DEL PROCESO DE INTEGRACIÓN ECONÓMICA DE AMÉRICA LATINA*
}

\author{
DYNAMICS AND DETERMINANTS OF THE LATIN AMERICAN ECONOMIC \\ INTEGRATION PROCESS \\ Luis Marcelo Florensa* \\ Laura Márquez-Ramos** \\ María Luisa Recalde*** \\ Pedro Gabriel Degiovanni****
}

enviado: 3 mayo 2017 - aceptado: 13 julio 2017

\begin{abstract}
Resumen
Este artículo estudia la dinámica del proceso de integración económica de América Latina y analiza cuáles han sido sus determinantes en distintos momentos del tiempo durante el período 1962-2009. Se estima un modelo de elección discreta en el que la variable explicada es la existencia de un acuerdo de integración entre dos países, mientras que las variables explicativas se clasifican en económicas, geográficas, políticas e institucionales. Las principales conclusiones determinan que los factores económicos y geográficos son importantes a la hora de explicar por qué los países firman acuerdos de integración comercial, pero su importancia es sensible al momento del tiempo considerado. Además, se encuentra que los factores políticos e institucionales juegan un papel relevante sobre la estrategia de integración.
\end{abstract}

- Florensa, L. M., Márquez-Ramos, L., Recalde, M. L., \& Degiovanni, P. G. (2018). La dinámica y los determinantes del proceso de integración económica de América Latina. Estudios económicos, 35 (70), 5-26. Agradecemos los comentarios de los dos evaluadores anónimos y del editor. También agradecemos los útiles comentarios de Pedro E. Moncarz.

* Instituto de Economía y Finanzas, Universidad Nacional de Córdoba, Argentina. Correo electrónico: florensa@eco.unc.edu.ar

** Institute for International Trade, Universidad de Adelaida, Australia; Departamento de Economía e Instituto de Economía Internacional, Universitat Jaume I, España. Correo electrónico: lmarquez@uji.es

*** Instituto de Economía y Finanzas, Universidad Nacional de Córdoba, Argentina. Correo electrónico:1recalde@eco.unc.edu.ar

**** Instituto de Economía y Finanzas, Universidad Nacional de Córdoba. 
Código JEL: F14, F15, F50.

Palabras clave: comercio internacional, acuerdos de integración económica, factores políticos e institucionales, Latinoamérica.

\begin{abstract}
This paper studies the dynamics of the economic integration process in Latin America and analyzes its determinants in different points of time during 1962-2009. A discrete choice model is estimated, in which the dependent variable is the existence of an integration agreement between two countries, while the explanatory variables are classified into economic, geographic, political and institutional factors. The main findings show that economic and geographical factors are important in explaining why countries sign trade integration agreements, although this importance is time sensitive. In addition, results suggest that political and institutional factors play an important role in integration strategies.
\end{abstract}

JEL Code: F14, F15, F50.

Keywords: International Trade; Economic Integration Agreements; Political and Institutional Factors, Latin America.

\title{
INTRODUCCIÓN
}

La firma de un acuerdo de integración comercial requiere de la toma de decisiones generalmente controvertidas debido a que genera beneficios globales que en ciertas ocasiones se distribuyen de manera desigual entre los países. Se pueden dar varios ejemplos de integración Norte-Norte, como es el caso de EE. UU. con Canadá; de acuerdos de integración Norte-Sur, como la posterior incorporación de México al Tratado de Libre Comercio de América del Norte (TLCAN); o ejemplos de acuerdos de integración Sur-Sur, como es el caso de la Asociación Latinoamericana de Integración (ALADI) y el Mercado Común del Sur (Mercosur). No obstante, la experiencia indica que las estrategias de integración europea y latinoamericana difieren y que el compromiso de constituir acuerdos de integración más profundos parece ser menor en Latinoamérica que en los países europeos (Florensa et al., 2015a) ${ }^{1}$. Asimismo, con el estancamiento de las nego-

1 Es importante, sin embargo, poner de relieve los hitos históricos que muestran el grado de desarrollo de las negociaciones comerciales entre las naciones en el nivel multilateral, desde la creación del GATT y su reemplazo más tarde por la OMC en la Ronda de Uruguay de 1995 (Delich et al., 2016). 
ciaciones comerciales multilaterales de la Ronda de Doha, se ha producido en la última década una proliferación de acuerdos de libre comercio a nivel bilateral, y ahora más recientemente grandes mega acuerdos comerciales que involucran a decenas de países y materias de negociación nuevas.

En este artículo se argumenta que hay aspectos políticos e institucionales que determinan la estrategia de integración económica y el grado de compromiso por parte de los distintos países latinoamericanos, en línea con estudios anteriores, como es el caso de Magee (2003), Márquez-Ramos et al. (2011) y Florensa et al. (2014b). En los primeros años del presente siglo, se ha discutido la existencia de dos estrategias de reconfiguración en la región. Por una parte, la estrategia del Pacífico reunía países integrados, en mayor medida, con economías de fuera de la región como Chile, Colombia, México y Perú con EE. UU. y la Unión Europea. La estrategia del Atlántico, en cambio, incluía países con un interés menor en la integración con mercados extrarregionales como la Argentina, Bolivia, Brasil, Ecuador y Venezuela. En este contexto, la elevada diversidad y heterogeneidad productiva de los países de la región, así como también de sus vínculos comerciales inter- e intrarregionales, convierte a la región latinoamericana en un caso altamente interesante para el análisis de la dinámica y la identificación de los determinantes de su proceso de integración económica.

El tema bajo estudio es también de especial relevancia desde el punto de vista de la política comercial pues la integración económica fomenta el comercio internacional (Florensa et al., 2015a; Márquez-Ramos et al., 2015), lo cual a su vez estimula el crecimiento económico de los países (Frankel y Romer, 1999). Por lo tanto, resulta de interés avanzar en el análisis de los factores que motivan que los países latinoamericanos firmen acuerdos de integración comercial con otros países de la región y del mundo. Esto ayudaría a una mejor comprensión de las consecuencias que tendrán los cambios políticos que se están experimentando actualmente en Latinoamérica² ${ }^{2}$.

2 Podría considerarse que el año 2016 supuso un punto de inflexión en algunos países de la región latinoamericana. Por ejemplo, en una reunión celebrada entre las presidentas de Chile y Brasil, se demostró el interés de Brasil en generar puentes entre el Mercosur y la Alianza del Pacífico (Montes, 2016). Otro ejemplo es que, con el cambio de gobierno de diciembre de 2015 en la Argentina, se vislumbró una nueva etapa en la política comercial y en la estrategia de inserción comercial internacional de este país. En el ámbito regional se destaca la importancia estratégica de las relaciones con Brasil, así como también la relevancia de la calidad jurídica de los compromisos asumidos (Peña, 2016). Según este autor se debe tener en cuenta la estrategia de adaptación del Mercosur a la realidad internacional y regional, articulando la Alianza del Pacífico, el Mercosur y el ALADI, así como también las negociaciones con otros espacios regionales. 
Este trabajo se compone de cinco partes. A continuación de la introducción se presenta una descripción de la evolución de los acuerdos de integración comercial en los que han participado los países del ALADI. En la tercera parte, se presenta la metodología utilizada y las hipótesis. Los resultados principales se describen en la cuarta parte $y$, finalmente, en la última parte se presentan las conclusiones.

\section{EVOLUCIÓN DE LOS ACUERDOS DE INTEGRACIÓN COMERCIAL}

Para la presente sección se utiliza la base de datos de acuerdos de integración económica disponible en la página web de Bergstrand para el período 19622009, ampliada con los acuerdos de integración notificados a la Organización Mundial del Comercio (OMC).

La figura 1 muestra la evolución del porcentaje de pares de países en el mundo que han firmado algún tipo de acuerdo comercial, mientras que la figura 2 muestra los acuerdos firmados por los once países fundadores del ALADI (Argentina, Bolivia, Brasil, Chile, Colombia, Ecuador, México, Paraguay, Perú, Uruguay, Venezuela) con otros países de la región y del resto del mundo.

Se distinguen cuatro niveles de profundización de los acuerdos: el nivel 1 incluye los acuerdos preferenciales de comercio no recíprocos (NRPTA por sus siglas en inglés); generalmente este tipo de acuerdo lo otorga un país desarrollado a otro de menor grado de desarrollo económico. El nivel 2 corresponde a los acuerdos preferenciales de comercio (o PTA). El nivel 3 corresponde a los acuerdos de libre comercio (o FTA) y el nivel 4 a las uniones aduaneras (o CU).

Las figuras 1 y 2 están construidas de manera que cada línea muestra el porcentaje de pares de países que poseen un acuerdo de integración económica (o AIE) de al menos la profundidad indicada. Por ejemplo, dentro de los países con acuerdos de libre comercio (es decir, cuando AIE es igual a 3) se consideran tanto los países con acuerdos de libre comercio como aquellos con uniones aduaneras.

Se observa que los patrones de integración a nivel mundial y para los 11 países del ALADI son muy similares. El mayor número de acuerdos se da en los niveles menos profundos de integración económica y hay un crecimiento pronunciado de los acuerdos hasta el nivel 3 (NRPTA, PTA y FTA) a partir de los años noventa. Si bien la tendencia difiere para cada tipo de acuerdo, en el caso de las uniones aduaneras se observa que hay un mayor porcentaje de países con este tipo de acuerdo en el mundo que en los países de ALADI. 
Figura 1. Evolución de los acuerdos comerciales a nivel mundial (\% de pares de países con acuerdo)

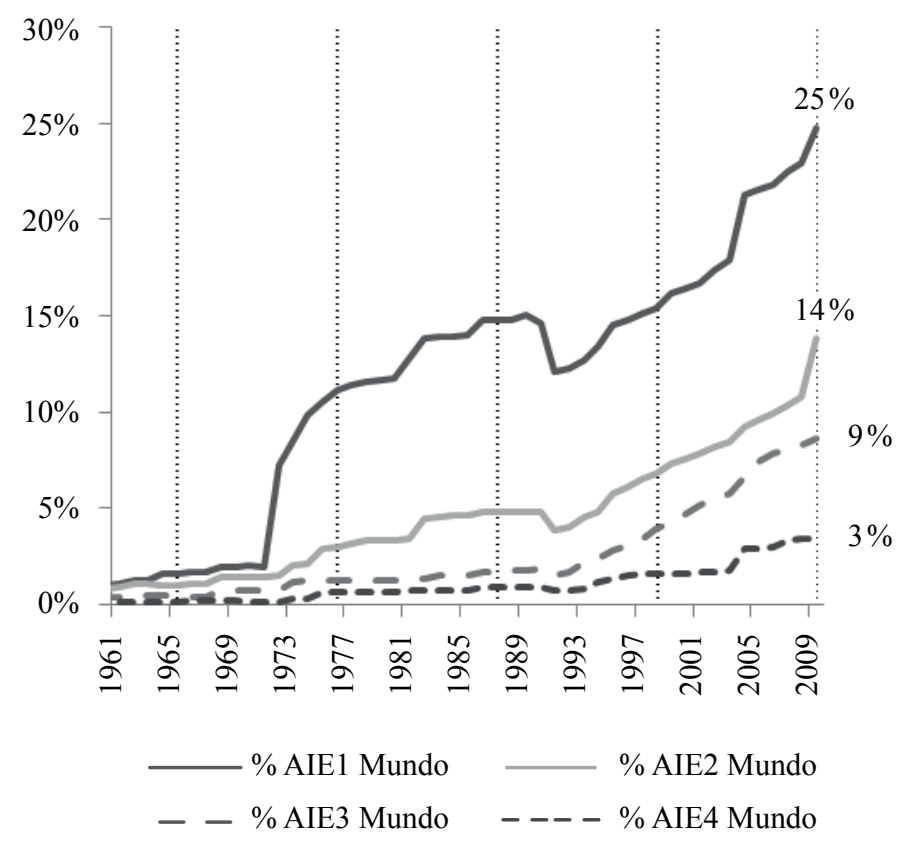

Fuente: elaboración propia a partir de los datos de Bergstrand (2015). AIEj hace referencia a los Acuerdos de Integración Económica de nivel mayor o igual a j (con j = 1, 2, 3, 4).

A principios de los noventa, los países de la región latinoamericana crearon uniones aduaneras (Mercosur y Comunidad Andina), aunque imperfectas; sin embargo, no hubo incrementos en esta categoría desde entonces. En contraste, el proceso de integración en este nivel de profundización ha continuado durante la década del 2000 cuando se tiene en cuenta a todo el mundo.

Los acuerdos de libre comercio han aumentado sostenidamente en el tiempo desde principios de los años noventa, como muestra la figura 1. Para el caso de América Latina, y como muestra la figura 2, hay un incremento en forma escalonada que corresponde a acuerdos que involucran a un gran número de países. Un ejemplo de ello es el acuerdo de libre comercio firmado entre la Unión Europea y Chile en 2003. 
Figura 2. Evolución de los acuerdos comerciales para los 11 países de ALADI (\% de pares de países con acuerdo)

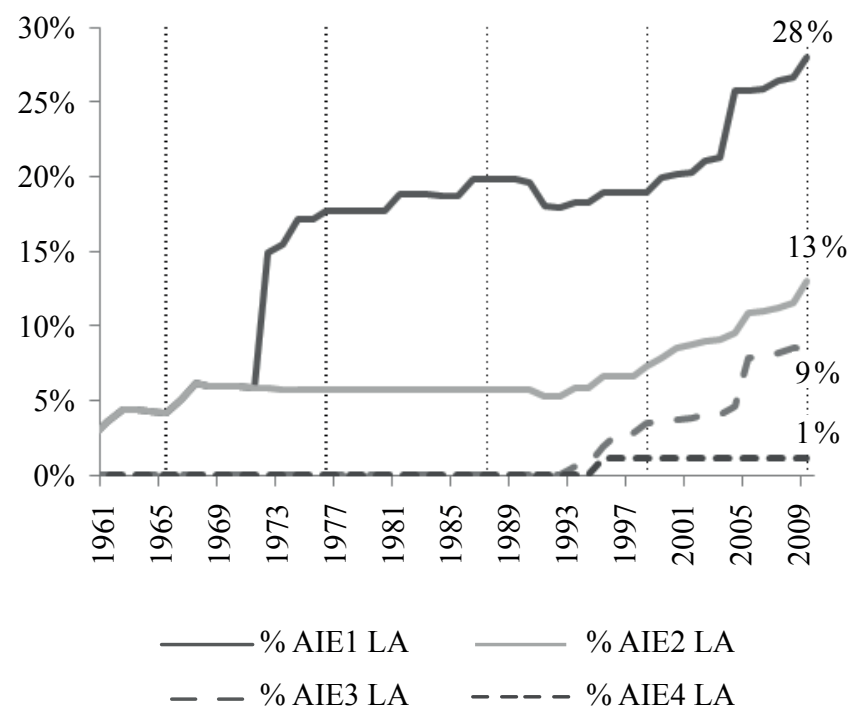

Fuente: elaboración propia a partir de los datos de Bergstrand (2015). AIEj hace referencia a los Acuerdos de Integración Económica de nivel mayor o igual a j (con j = 1, 2, 3, 4).

En cuanto a los acuerdos preferenciales, Latinoamérica tuvo históricamente una mayor proporción que el mundo, debido a la temprana conformación de ALALC, ALADI y la Comunidad Andina. Sin embargo, cuando se considera a todos los países del mundo, aumenta paulatinamente la participación relativa de los acuerdos y, en 2008, con la firma entre la Unión Europea y el CARIFORUM, la proporción del mundo supera a la de los países de América Latina.

En cuanto a los NRPTA, se observa una primera etapa de gran incremento durante los años setenta. La importancia relativa de este tipo de acuerdo disminuye a fines de los ochenta ${ }^{3}$, para volver a incrementarse a partir de los noventa de manera progresiva. Se observa un aumento significativo en el año 2004 para el mundo y para Latinoamérica, que responden a la importante expansión de la Unión

3 Esta caída se debe al desmembramiento de los países soviéticos, por lo que aumenta el número de países que no tienen acuerdos. 
Europea, que extendió el número de países que otorgan acuerdos preferenciales no recíprocos a los países en desarrollo.

A pesar de comenzar relativamente temprano su proceso de integración, la escasa coordinación existente en América Latina ha llevado a que en la actualidad se cuente de manera simultánea con al menos ocho acuerdos de integración y/o vinculación intrarregional: la Unión de Naciones Suramericanas (Unasur), el Mercosur, la Comunidad Andina, el ALADI, el Sistema Económico Latinoamericano y del Caribe (SELA), la Alianza Bolivariana para América o ALBA, la Alianza del Pacífico y la Comunidad de Estados Latinoamericanos y Caribeños (Celac).

\section{ANÁLISIS ECONOMÉTRICO}

\section{II.1. Especificación del modelo}

En línea con Baier y Bergstrand (2004), el análisis econométrico se basa en un modelo de elección discreta, donde $\mathrm{y}^{*}$ es una variable no observada (o latente) que representa la diferencia en los niveles de utilidad de firmar un acuerdo de integración económica 4 :

$$
y^{*}=a+x^{\prime} b+e,
$$

en el cual $\mathrm{x}$ es un vector de variables explicativas, $\mathrm{b}$ es un vector de parámetros, $\mathrm{y}$ e es el término de error, que se supone independiente de $\mathrm{x} y$ que sigue una distribución logística ${ }^{5}$.

Puesto que $\mathrm{y}^{\star}$ es inobservable, se define una variable, EIA, que es igual a 1 si los países $\mathrm{i}, \mathrm{j}$ tienen algún tipo de acuerdo de integración económica (indicando que $\mathrm{y}^{\star}$ es mayor que 0), y 0 si no lo tienen (indicando que $\mathrm{y}^{\star}$ es menor o igual a 0 ).

La probabilidad de respuesta es:

$$
P(A I E=1)=P\left(y^{*}>0\right)=G\left(a+x^{\prime} b\right),
$$

4 Por simplicidad, no se incluyen los subíndices correspondientes a cada observación.
5 El modelo probit sigue una distribución normal estándar. 
en la cual $\mathrm{G}(\bullet)$ es la función acumulada de la distribución logística, que asegura que la probabilidad de que exista un acuerdo, es decir que $\mathrm{P}(\mathrm{AIE}=1)$ se encuentre entre 0 y $1^{6}$.

Estimando este modelo, se obtiene información de la dirección del efecto (efecto positivo o negativo) y la significatividad estadística de las variables explicativas sobre la probabilidad de que exista un acuerdo de integración económica (véase, por ejemplo, Wooldridge, 2008). Puesto que en este artículo se trata de responder a las preguntas: 1) ¿son relevantes los factores geográficos, económicos, políticos e institucionales a la hora de firmar un acuerdo en América Latina?, y 2) ¿cuál es la dirección del efecto?, se utilizan dos ecuaciones de regresión. La primera considera todo el período 1962-2009 y la segunda es una regresión para un año seleccionado en cada década, partiendo desde la década del setenta. Los años seleccionados son 1976, 1987, 1998 y $2009^{7}$.

Finalmente, y siguiendo la literatura relacionada (Márquez-Ramos et al., 2011), se incluye la variable acuerdos de integración económica rezagada en la especificación que considera todo el período. Esta variable estaría indicando el tipo de integración económica alcanzado en años anteriores y toma el valor 0 si no había acuerdo y los valores 1-4 si había acuerdo (en función del grado de profundización alcanzado en el acuerdo de integración, véase sección I). De manera que si, por ejemplo, los países $i, j$ tienen un acuerdo anterior es probable que en años sucesivos lo tengan también.

Se estima un modelo de regresión logística (logit) donde la variable dependiente es la existencia de un acuerdo y toma el valor uno si existe acuerdo entre uno de los once países de ALADI i y cualquier otro país del mundo j. Las variables explicativas consideradas incluyen factores económicos, geográficos, políticos e institucionales.

6 El marco econométrico que se emplea es el Modelo de Elección Cualitativa de McFadden (1975, 1976). En él se tiene una variable no observable (latente) $y^{*}$ que en este contexto representa la diferencia de utilidad resultante de tomar una determinada acción que, en nuestro caso, es la formación de un AIE. Lo que efectivamente se analiza es la probabilidad de que dos países firmen un acuerdo, ya que la variable latente $y^{*}$ se relaciona con una variable aleatoria Bernoulli $y$ que representa la existencia o no de un AIE. La relación entre ambas variables viene dada por la ecuación (2).

7 Estos años fueron seleccionados teniendo en cuenta la mayor disponibilidad posible de observaciones para realizar las regresiones. No se seleccionó ningún año de la década que comienza en 1960 porque no existen datos de comercio antes de 1962 y se trabaja con algunas variables rezagadas 10 años para evitar un posible problema de endogeneidad de las variables independientes de interés. 
En concreto, se utilizan el PIB real, diferencia del PIB y comercio bilateral entre $\mathrm{i}, \mathrm{j}$ (variables económicas); una variable relacionada con la distancia geográfica que denominamos "natural" (en el sentido de si dos países son "socios naturales"), remoticidad, si los países tienen o no salida al mar y si comparten frontera común (variables geográficas); los derechos políticos y el grado de democracia alcanzado (variables políticas e institucionales). Además, se considera un regresor adicional que controla por la similitud cultural entre los países i, j (si comparten un mismo lenguaje).

Baier y Bergstrand (2004) demuestran que la ganancia de bienestar neta que obtiene un par de países que firman un acuerdo de integración económica aumenta a medida que la distancia entre ellos disminuye, la remoticidad respecto del resto del mundo aumenta y cuanto mayor y más similar es el tamaño de sus economías. Por lo tanto se espera que los coeficientes estimados asociados a las variables PIB real, natural ${ }^{8}$ y remoticidad sean positivos, mientras el asociado a la diferencia entre los PIB sea negativo. Además, se espera que mayor comercio internacional entre dos socios, que alguno de ellos no tenga salida al mar o que compartan frontera, conlleve a que la probabilidad de firmar un acuerdo sea mayor (véase Magee, 2003; Baier y Bergstrand, 2004; Márquez-Ramos et al., 2011).

En cuanto a las variables políticas e institucionales, como el valor de la variable "derechos políticos" aumenta a medida que dichos derechos disminuyen, mientras que el valor del "grado de democracia" aumenta con el nivel democrático imperante, se espera que el coeficiente estimado para la primera variable tenga signo negativo y el de la segunda, signo positivo.

La muestra incluye 161 países para el período 1962-2009; con lo cual se tiene un total de 45.304 observaciones asociadas a 1700 pares de países. Es necesario aclarar que no todas las variables están disponibles para todas las observaciones que constituyen la muestra, razón por la cual el número de observaciones viables para llevar a cabo las regresiones resulta ser menor.

Los estadísticos descriptivos para las variables se presentan en la tabla 1 y los detalles sobre las fuentes y la construcción de las variables se encuentran en la tabla A.1 del apéndice. Finalmente, la tabla A.2 del apéndice incluye los acuerdos que han firmado los países de la región incluidos en el análisis y complementa el análisis econométrico con una visión más descriptiva. En concreto, esta tabla ayu-

8 La variable "natural" se define como el logaritmo natural de la inversa de la distancia entre los socios comerciales, por lo tanto, a menor distancia, mayor es el valor de esta variable. 
da a ver la diferencia entre el enfoque que han seguido países como la Argentina y Brasil, por un lado, y Chile y México, por el otro, diferencia que se analiza mediante la estimación del modelo econométrico.

Tabla 1. Estadísticos descriptivos

\begin{tabular}{lccccc}
\hline Variable & Observ. & Media & Desv. Est. & Min & Max \\
\hline $\begin{array}{l}\text { AIE (variable } \\
\text { dependiente) }\end{array}$ & 45.304 & 0,32 & 0,47 & 0 & 1 \\
PIB real & 39.193 & 49,36 & 2,49 & 40,20 & 57,86 \\
Dif. PIB & 39.193 & 2,41 & 1,68 & 0 & 9,21 \\
Natural & 44.234 & $-8,96$ & 0,71 & $-9,89$ & $-5,37$ \\
Remoticidad & 44.234 & 2,61 & 4,15 & 0 & 9,28 \\
Sin salida al mar & 44.234 & 0,20 & 0,4 & 0 & 1 \\
Frontera común & 44.234 & 0,04 & 0,21 & 0 & 1 \\
Mismo lenguaje & 44234 & 0,18 & 0,38 & 0 & 1 \\
Der. Politicos & 33.635 & 9,99 & 9,04 & 1 & 49 \\
Gr. Democracia & 33.336 & 7,81 & 10,11 & -19 & 20 \\
Comercio & 45.303 & 8 & 3,36 & $-6,91$ & 19,22 \\
\hline
\end{tabular}

Fuente: elaboración propia.

\section{2. Hipótesis}

Las principales hipótesis que se contrastan son las siguientes.

En primer lugar, que los factores económicos y geográficos son importantes para explicar por qué los países firman acuerdos de integración comercial, pero que su importancia puede ser sensible al momento del tiempo considerado.

Como segunda hipótesis se plantea que los factores políticos e institucionales son relevantes pero que también pueden depender del período. En este sentido, 
conviene aclarar que a principios del período analizado hubo una mayor presencia de regímenes no democráticos en América Latina; en la actualidad, todos estos países tienen democracias. Una vez que la democracia se ha consolidado, lo importante es que el régimen democrático no sea solamente formal sino que esté acompañado de una serie de factores y circunstancias que permitan el buen funcionamiento de las instituciones. Por tanto, se espera que la variable "grado de democracia" haya sido más importante en los años 70-80 mientras que la variable “derechos políticos" lo sea en los años 90-2000. Lo anterior pone de manifiesto la importancia que tiene el proceso de consolidación democrática como un requisito para que los países se integren al mundo.

\section{RESULTADOS}

La tabla 2 muestra los resultados de la estimación utilizando el modelo logit. Debajo de los coeficientes estimados, se encuentran los estadísticos z y, debajo de estos, están los efectos marginales, resaltados en letra itálica, obtenidos a nivel de su valor medio. Estos últimos brindan información sobre el efecto ceteris paribus de cada variable independiente sobre la probabilidad de que la variable dependiente tome el valor 1 .

La columna (1) contiene los resultados correspondientes a estimar la regresión utilizando todas las observaciones del período 1962-2009, mientras que las columnas (2) a (5) contienen los valores correspondientes a las regresiones para 1976, 1987, 1998 y 2009.

La estimación para todo el período incluye dummies temporales ${ }^{9}$ y una variable que mide el grado de profundización de los acuerdos de comercio bilaterales ${ }^{10}$. Los coeficientes asociados a las variables económicas y geográficas, para esta regresión, tienen el signo esperado y son estadísticamente significativos, excepto la variable "natural" que no es significativa. Con respecto a la variable "mismo lenguaje", los resultados prueban que, para los países latinoamericanos de la muestra,

9 Específicamente, se incluye una variable ficticia que toma el valor uno en cada año determinado y cero en caso contrario, excluyendo un año para evitar problemas de multicolinealidad perfecta.

10 La liberalización comercial afecta con retardo el volumen de comercio y este a su vez influye en la asignación de la dotación de factores y el ingreso. Por lo tanto, dichas variables pueden ser endógenas. Una forma de evitar la endogeneidad es considerarlas, pero con un rezago temporal. Baier y Bergstrand (2007) muestran que el impacto pleno de la firma de un AIE sobre el comercio se produce al cabo de los 10 años. Por este motivo, las variables rezagadas fueron introducidas en el modelo con un rezago de 10 años. 
tener un mismo lenguaje no es un factor determinante a la hora de firmar acuerdos de integración comercial, al menos cuando ya se controla por el efecto "frontera común" en la especificación ${ }^{11}$. Según los resultados obtenidos, un mayor grado de democracia en los países implicados hace que sea más probable que se firme un acuerdo entre ellos. La variable "derechos políticos" está medida de tal forma que se espera que su coeficiente sea negativo y es esto lo que se obtiene encontrándose, por tanto, evidencia a favor de que mayores derechos políticos incrementan la probabilidad de crear nuevos acuerdos de integración regional.

La variable de comercio rezagada ejerce una influencia directa y significativa sobre la existencia y creación de acuerdos de integración. Esto se traduce en que, a mayor comercio en años anteriores, más probable será que se creen y se mantengan los acuerdos en el tiempo. Por último, en esta primera regresión que incluye todos los años del período 1962-2009, la variable de acuerdos de integración económica (AIE) rezagada es significativa y su coeficiente es positivo, lo cual indica que una mayor profundización de los acuerdos anteriores hace que los mismos perduren en el tiempo.

La segunda columna de la tabla 2 muestra los resultados obtenidos para 1976. En este caso, se aprecian dos características de interés. En primer lugar, la variable "natural" tiene un efecto contrario al esperado, pues su coeficiente es negativo y estadísticamente significativo. Esto se debe, posiblemente, a que en la década de los setenta hubo una proliferación de acuerdos entre países de América Latina y países desarrollados que geográficamente están lejos. Lo anterior queda reflejado en el salto pronunciado de la línea AIE1 (NRPTA) de la figura 2 en este período y en la tabla A.2 del apéndice, donde se listan los nuevos acuerdos de la región por período. La segunda característica que se observa es que la variable "derechos políticos" no es estadísticamente significativa. Lo mismo sucede al analizar el año 1987 (columna 3 de la tabla 2). Esto está en línea con la hipótesis planteada de que los factores políticos e institucionales dependen del período considerado. Habíamos señalado que cuando los países no son democráticos, lo importante es que se transformen en democracias. Una vez se consolidan, es deseable que comiencen a estar acompañadas de un buen funcionamiento institucional. Por tanto, es de esperar que la variable "derechos políticos" incremente su importancia en los períodos de tiempo más recientes. Esto es consistente con los resultados obtenidos: para los años 1976 y 1987, la variable "grado de democracia" ejerce una influencia significa-

11 Para testear si las variables que resultaron no significativas, de manera individual, lo son de manera conjunta, se llevó a cabo un Test de Razón de Verosimilitud. El resultado obtenido conduce a rechazar la hipótesis nula en la regresión realizada para todo el período. 
tiva sobre la probabilidad de que se creen o de que existan acuerdos de integración; sin embargo, en los años 1998 y 2009, esta variable no es estadísticamente significativa. Por el contrario, la variable "derechos políticos" no es significativa en los años 1976 y 1987, pero sí lo es, y su coeficiente estimado tiene el signo esperado, en los años 1998 y 2009.

Finalmente, en los resultados de la columna 5, que incluyen los del año más reciente que se considera en este análisis (2009), las variables geográficas de remoticidad y frontera común pierden significatividad. En concreto, se observa que en el año 2009 estas variables no son estadísticamente significativas. Parece, por tanto, que los acuerdos de integración más recientes ya no se ven tan condicionados por factores geográficos como en períodos anteriores. Esto podría deberse, en parte, a la disminución de los costos de transporte como consecuencia de las mejoras tecnológicas ocurridas en los últimos años.

También se estimaron los parámetros del modelo utilizando una regresión probit. Ambas estimaciones, logit y probit, resultan similares, lo cual avala las hipótesis planteadas ${ }^{12}$.

Tabla 2. Resultados de la regresión (logit)

\begin{tabular}{|c|c|c|c|c|c|}
\hline & & & & & \\
\hline & Todo (1) & $1976(2)$ & $1987(3)$ & 1998 (4) & 2009 (5) \\
\hline \multirow[t]{3}{*}{ PIB real (rez.) } & $0,247^{\star * *}$ & $0,477^{\star \star \star}$ & $0,462^{\star \star *}$ & $0,615^{\star \star \star}$ & $0,443^{\star * *}$ \\
\hline & 11,832 & 4,411 & 5,200 & 6,929 & 7,104 \\
\hline & 0,016 & 0,119 & 0,112 & 0,146 & 0,110 \\
\hline \multirow[t]{3}{*}{ Dif. PIB (rez.) } & $-0,099^{\star * *}$ & $-0,228^{\star *}$ & $-0,355^{\star * *}$ & $-0,330^{* * *}$ & $-0,196^{\star * *}$ \\
\hline & $-5,398$ & $-2,247$ & $-3,951$ & $-3,146$ & $-3,405$ \\
\hline & $-0,006$ & $-0,057$ & $-0,086$ & $-0,078$ & $-0,049$ \\
\hline \multirow[t]{3}{*}{ Natural } & 0,124 & $-1,044^{\star * *}$ & $-0,388$ & 0,161 & $1,213^{\star * *}$ \\
\hline & 1,598 & $-2,861$ & $-1,092$ & 0,427 & 3,654 \\
\hline & 0,008 & $-0,261$ & $-0,094$ & 0,038 & 0,302 \\
\hline \multirow[t]{2}{*}{ Remoticidad } & $0,039^{* * *}$ & $0,450^{\star * *}$ & $0,133^{\star *}$ & $0,208^{\star * *}$ & $-0,022$ \\
\hline & 3,220 & 6,259 & 2,252 & 3,104 & $-0,467$ \\
\hline
\end{tabular}

12 Los resultados de la regresión utilizando el Método Probit se encuentran a disposición de quienes los soliciten. 
ESTUDIOS ECONOMICOS

\begin{tabular}{|c|c|c|c|c|c|}
\hline & 0,002 & 0,113 & 0,032 & 0,049 & $-0,005$ \\
\hline \multirow[t]{3}{*}{ Sin salida mar } & $2,254^{\star * *}$ & $4,554^{\star \star \star}$ & $5,433^{\star * *}$ & $4,213^{\star * \star}$ & $2,318^{\star * *}$ \\
\hline & 28,357 & 9,136 & 8,794 & 7,234 & 9,194 \\
\hline & 0,143 & 1,138 & 1,314 & 1,00 & 0,578 \\
\hline \multirow[t]{3}{*}{ Frontera común } & $1,313^{\star * *}$ & $2,584^{\star \star \star}$ & $1,830^{\star * *}$ & $2,705^{\star * *}$ & 0,924 \\
\hline & 6,577 & 3,004 & 2,909 & 3,153 & 1,153 \\
\hline & 0,083 & 0,646 & 0,442 & 0,643 & 0,230 \\
\hline \multirow[t]{3}{*}{ Mismo lenguaje } & $-0,002$ & $-0,735$ & 0,624 & 0,001 & 0,103 \\
\hline & $-0,015$ & $-1,403$ & 1,374 & 0,003 & 0,288 \\
\hline & $-0,000$ & $-0,184$ & 0,151 & 0,00 & 0,025 \\
\hline \multirow[t]{3}{*}{ Der. Políticos } & $-0,057^{\star * *}$ & $-0,006$ & $-0,112$ & $-0,431^{\star * *}$ & $-0,227^{\star * x}$ \\
\hline & $-6,286$ & $-0,239$ & $-1,581$ & $-5,597$ & $-4,418$ \\
\hline & $-0,004$ & $-0,001$ & $-0,127$ & $-0,102$ & $-0,057$ \\
\hline \multirow[t]{3}{*}{ Gr.Democracia } & $0,060^{* * *}$ & $0,056^{\star *}$ & $0,117^{\star * *}$ & $-0,008$ & 0,038 \\
\hline & 9,743 & 2,117 & 3,189 & $-0,144$ & 1,419 \\
\hline & 0,004 & 0,014 & 0,028 & $-0,002$ & 0,009 \\
\hline \multirow[t]{3}{*}{ Comercio (rez) } & $0,216^{\star * *}$ & $0,475^{\star \star \star}$ & $0,340^{* * *}$ & $0,276^{\star * *}$ & $0,141^{\star * *}$ \\
\hline & 15,358 & 6,516 & 4,997 & 3,568 & 2,676 \\
\hline & 0,014 & 0,119 & 0,082 & 0,065 & 0,035 \\
\hline
\end{tabular}

AIE (rez.)

$7,287^{\star * *}$

25,182

0,461

Constante

$-15,412^{\star * *}$

$-37,113^{\star * *}$

$29,936^{\star * *}$

$29,734^{\star * *}$

$-11,635^{\star * *}$

$-13,122$

$-6,385$

$-5,428$

$-5,464$

$-3,018$

Observaciones

22155

519

526

622

849

Log. Verosim.

$-4664$

$-1839$

$-1820$

$-1927$

$-3515$

Pseudo R2

0,696

0,489

0,497

0,553

0,402

$\mathrm{p}<0,10 ;{ }^{* *} \mathrm{p}<0,05 ;{ }^{* *} \mathrm{p}<0,01$. Debajo de los coeficientes se presentan los estadísticos $\mathrm{Z}$ (robustos) y debajo de los mismos los efectos marginales. Los rezagos de las variables son para 10 años. 


\section{CONCLUSIONES}

Este artículo estima un modelo de elección discreta en el cual la variable explicada es la existencia de acuerdos de integración entre dos países, al menos uno de ellos de Latinoamérica, mientras que las variables explicativas se clasifican en económicas, geográficas, políticas e institucionales. Se contrastaron dos hipótesis: 1) los factores económicos y geográficos son importantes para explicar por qué los países firman acuerdos de integración comercial y 2) los factores políticos e institucionales son relevantes. La presente investigación resulta de interés en un contexto de la consolidación de cadenas globales de valor que han reconfigurado el comercio internacional en años recientes ${ }^{13}$.

Los resultados encontrados avalan las hipótesis propuestas e indican que los factores económicos y geográficos son importantes cuando se quiere explicar por qué los países firman acuerdos de integración comercial. Además, se demuestra que el grado de democracia y los derechos políticos también han jugado un rol importante sobre la estrategia de integración en Latinoamérica.

De acuerdo con los resultados obtenidos en este estudio, los cambios que se vislumbran en la política económica y en el fortalecimiento institucional en algunos de los países latinoamericanos podrían derivar en un incremento en el número de nuevos acuerdos de integración económica. La creación de nuevos acuerdos podría no derivar en el modelo de apertura al mundo esperado, y en el consiguiente aumento del comercio internacional y del crecimiento económico, si no se tiene en cuenta la necesidad de una correcta coordinación en materia de integración económica en la región.

13 Para un análisis sobre la producción en red de América Latina, véase Florensa et al, 2015b. 


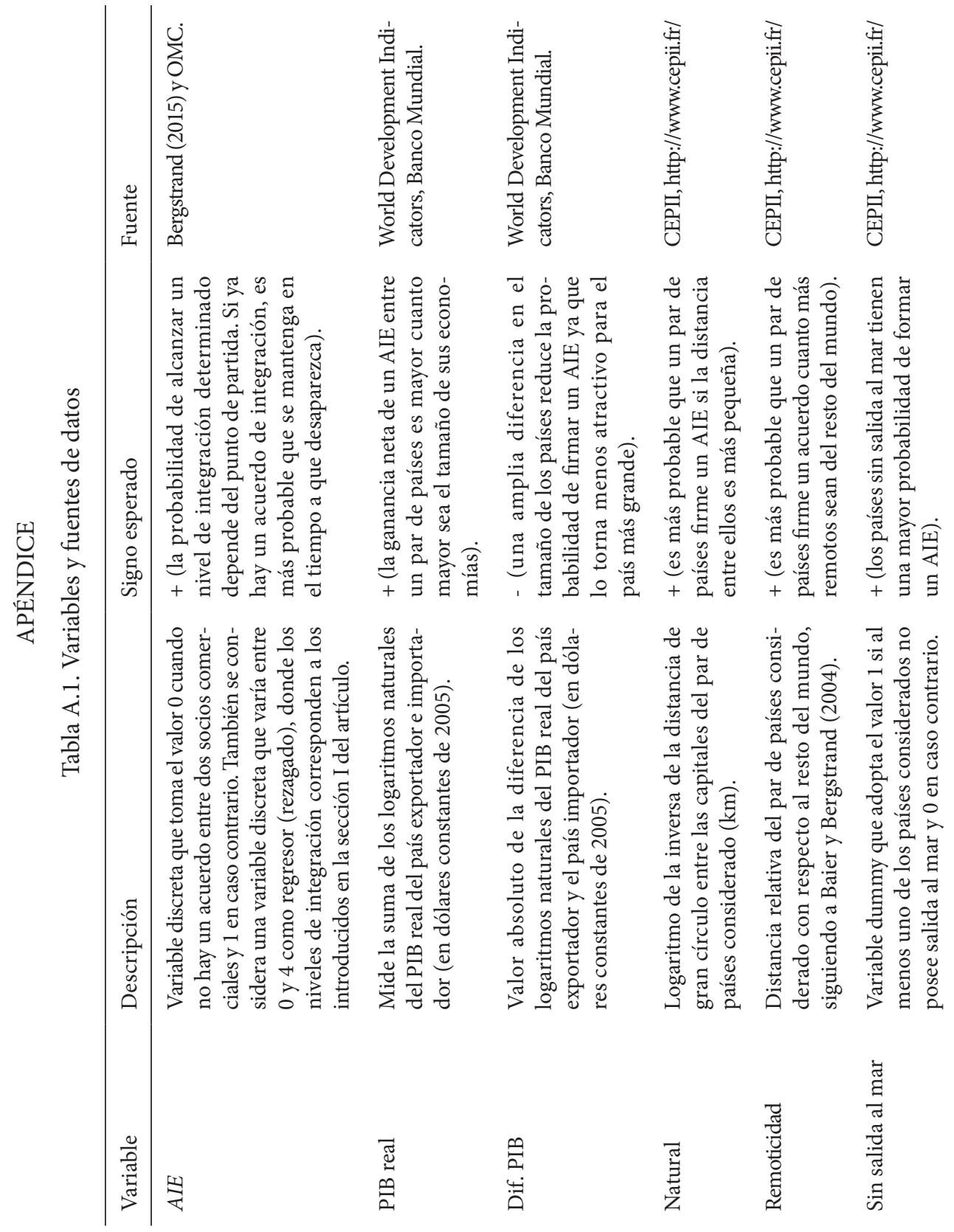


(1)

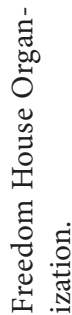

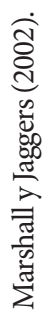

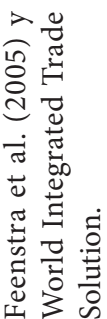

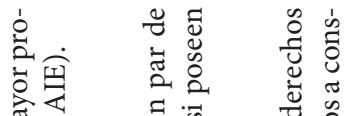

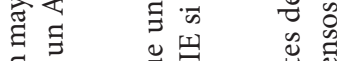

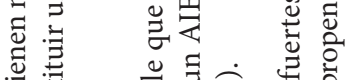

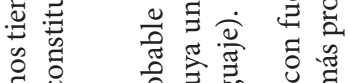

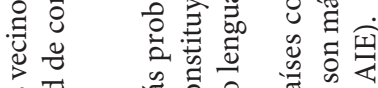

芯

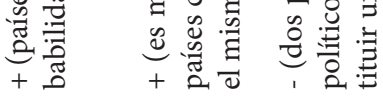

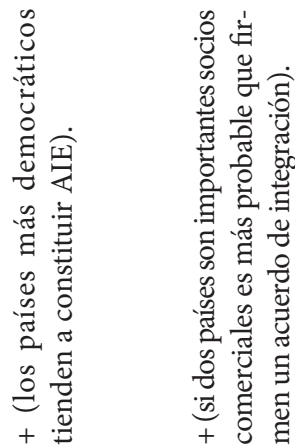

-

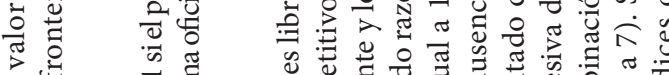

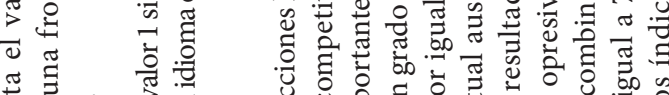

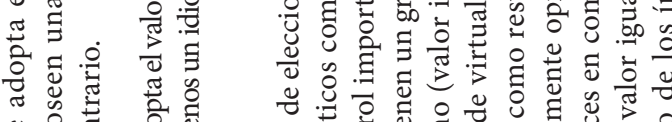

o

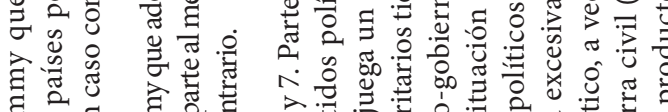

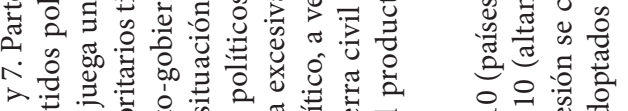

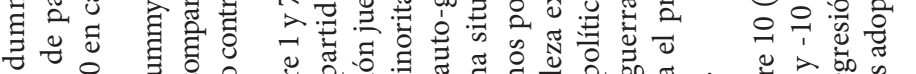

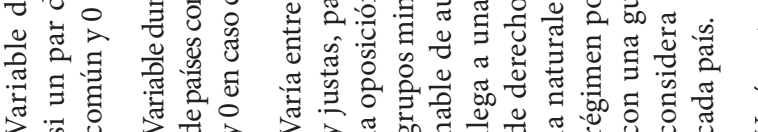

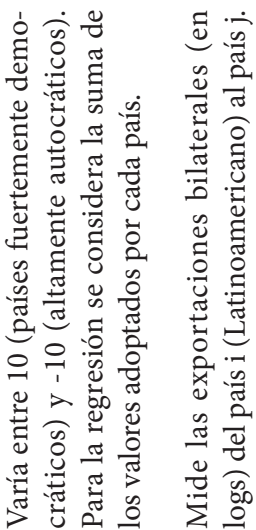

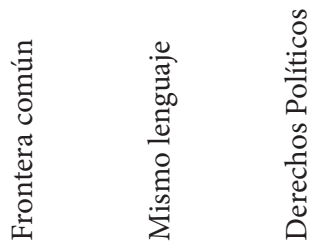

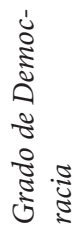

$\frac{0}{3}$ 
Tabla A.2.

\section{Acuerdos vigentes}

\section{Acuerdos vigentes firmados en la década 1960-1969}

-Asociación Latinoamericana de Libre Comercio - ALALC (1960), compuesta por Argentina, Bolivia, Brasil, Chile, México, Paraguay, Perú y Uruguay.

\section{Acuerdos vigentes firmados en la década 1970-1979}

-Acuerdos Preferenciales de Comercio No Recíprocos

- Australia (1974)

- Canadá (1974)

- Estados Unidos (1976)

- Japón (1971)

- Liechtenstein (1972)

- Noruega (1971)

- Nueva Zelanda (1972)

- Suiza (1972)

- Unión Europea (1971)

\section{- Acuerdos Preferenciales de Comercio}

-Comunidad Andina - CAN (1979), compuesta por Bolivia, Colombia, Ecuador, Perú y Venezuela ${ }^{14}$.

\section{Acuerdos vigentes firmados en la década 1980-1989}

\section{- Acuerdos Preferenciales de Comercio}

-Asociación Latinoamericana de Integración - ALADI (1981), compuesta por Argentina, Bolivia, Brasil, Chile, Colombia, Ecuador, México, Paraguay, Perú, Uruguay y Venezuela.

- Acuerdos de Libre Comercio

- Venezuela-Guatemala (1987)

14 Chile se retiró de la CAN en 1976. Venezuela la abandonó en 2006. 


\section{Acuerdos vigentes firmados en la década 1990-1999}

- Acuerdos Preferenciales de Comercio No Recíprocos

- Sistema generalizado de preferencias de Rusia (1994)

- Acuerdos Preferenciales de Comercio

- Colombia-CARICOM ${ }^{15}$ (1995)

- Venezuela-CARICOM (1993)

- Acuerdos de Libre Comercio

- Bolivia -Chile (1993)

- Bolivia-México (1995)

- Chile-Canadá (1997)

- Chile-MERCOSUR (1996)

- Chile-Perú (1998)

- Chile-Venezuela (1993)

- Colombia-México (1995)

- México-Costa Rica (1995)

- México - Nicaragua (1998)

Tratado de Libre Comercio de América del Norte - TLCAN (1995), conformado por México, Canadá y Estados Unidos.

\section{- Uniones Aduaneras}

- Comunidad Andina - CAN (1995), compuesta por Bolivia, Colombia, Ecuador, Perú y Venezuela. Incrementa la profundidad de su integración. ${ }^{16}$

- Mercado Común del Sur - MERCOSUR (1991), compuesto por Argentina, Brasil, Paraguay y Uruguay.

\section{Acuerdos vigentes firmados en la década 2000-2009}

- Acuerdos Preferenciales de Comercio No Recíprocos

- Bielorrusia (2004)

15 CARICOM está compuesta por Antigua y Barbuda, Bahamas, Barbados, Belice, Dominica, Granada, Guyana, Jamaica, Montserrat, Saint Lucia, St. Kitts y Nevis, St. Vicente y las Granadinas, Surinam y Trinidad y Tobago.

16 Venezuela abandonó la CAN en 2006. 
- Islandia (2000)

- Turquía (2002)

- Acuerdos Preferenciales de Comercio

- Ingreso de Cuba a la ALADI (1999)

- Acuerdos de Libre Comercio

- Acuerdo Transpacífico de Integración Económica (2007), compuesto por Chile, Brunei, Nueva Zelanda y Singapur.

- CAN-MERCOSUR (2005)

- Chile-AELC1 (2004)

- Chile-China (2007)

- Chile-Colombia (2009)

- Chile-Corea del Sur (2004)

- Chile-Costa Rica (2002)

- Chile-El Salvador (2002)

- Chile-EE. UU. (2004)

- Chile-Honduras (2008)

- Chile-India (2008)

- Chile - Japón (2008)

- Chile - MCCA (2002)17

- Chile-México (1999)

- Chile-Panamá (2009)

- Chile-Unión Europea (2003)

- México-AELC (2001) ${ }^{18}$

- México-Israel (2000)

- México-Japón (2005)

- México-Triángulo Norte de Centroamérica (2001)19

- México-Unión Europea (2000)

- Perú-EE. UU. (2009)

17 El Mercado Común Centroamericano está compuesto por Costa Rica, El Salvador, Guatemala, Honduras y Nicaragua.

18 La Asociación Europea de Libre Comercio está compuesta por Islandia, Liechtenstein, Noruega y Suiza.

19 Conformado por El Salvador, Guatemala y Honduras. 


\section{REFERENCIAS BIBLIOGRÁFICAS}

Baier S. L., \& Bergstrand, J. H. (2004). Economic Determinants of Free Trade Agreements. Journal of International Economics, 64 (1), 29-63.

Baier, S. L., \& Bergstrand, J. H. (2007). Do free trade agreements actually increase members' international trade? Journal of International Economics, 71 (1), 72-95.

Bergstrand, J. H. (2015). Database on Economic Integration Agreements (September 2015). Disponible en http://www3.nd.edu/ jbergstr/

Delich, V., López, D., \& Muñoz, F. (ed.) (2016). 20 años de la OMC: una perspectiva desde Latinoamérica. Buenos Aires: FLACSO. Sede Académica Argentina, Programa de Cátedras OMC, Universidad de Chile.

Feenstra, R., Lipsey, R., Deng, H., Ma, A., \& Mo, H. (2005). World Trade Flows: 19622000. National Bureau of Economic Research, Working Paper No. 11040.

Florensa, L. M., Márquez-Ramos, L., Recalde, M. L., \& Barone, M. V. (2014a). Does Economic integration increase trade margins? Empirical evidence from LAIAs countries. Universitat Jaume I. Working Paper No. 2014/05.

Florensa, L. M., Márquez-Ramos, L., \& Recalde, M. L. (2014b). Revisiting the role of institutional and political factors on economic integration. The case of Latin America. Artículo presentado en las Jornadas de Economía del Banco Central del Uruguay.

Florensa, L. M., Márquez-Ramos, L., \& Recalde, M. L. (2015a). The effect of economic integration and institutional quality of trade agreements on trade margins: evidence for Latin America. Review of World Economics, 151 (2), 329-351.

Florensa, L. M., Márquez-Ramos, L., Martínez-Zarzoso, I., \& Recalde, M. L. (2015b). Regional versus global production networks: where does Latin America stand? Applied Economics, 47 (37), 3938-3956.

Frankel, J. A., \& Romer, D. H. (1999). Does trade cause growth? American Economic Review, 89 (3), 379-399.

Magee, C. S. (2003). Endogenous preferential trade agreements: An empirical analysis. Contributions in Economic Analysis \& Policy, 2 (1), 1-19.

Márquez-Ramos, L., Martínez-Zarzoso, I., \& Suárez-Burguet, C. (2011). Determinants of Deep Integration: Examining Socio-political Factors. Open Economies Review, 22 (3), 479-500.

Márquez-Ramos, L., Florensa, L. M., \& Recalde, M. L. (2015). Economic Integration Effects on Trade Margins: Sectoral Evidence from Latin America. Journal of Economic Integration, 30 (2), 269-299.

Marshall, M., \& Jaggers, K. (2002). Polity IV Project: Dataset Users' Manual. Estados Unidos: Universidad de Maryland. 
McFadden, D. (1975). The revealed preferences of a government bureaucracy: theory. Bell Journal of Economics, 6 (2), 401-416.

McFadden, D. (1976). Quantal choice analysis: a survey. Annals of Economic and Social Measurement, 5 (4), 363-390.

Montes, R. (26/02/2016). Rousseff y Bachelet pactan medidas económicas en plena crisis política, El País. Recuperado de http://internacional.elpais.com/internacional/2016/02/26/actualidad/1456524478_937366.html

Peña, F. (febrero, 2016). Política comercial e integración regional de Argentina: ¿hacia una nueva etapa? Puentes 17 (1). https://www.ictsd.org/bridgesnews/puentes/news/pol\%C3\%ADtica-comercial-e-integraci\%C3\%B3nregional-de-argentina-\%C2\%BFhacia-una-nueva

Wooldridge, J. M. (2008). Introductory Econometrics: A Modern Approach. Mason, Ohio: Cengage Learning.

(C) 2018 por los autores; licencia otorgada a la revista Estudios económicos. Este artículo es de acceso abierto y distribuido bajo los términos y condiciones de una licencia Atribución-No Comercial 3.0 Unported (CC BY-NC 3.0) de Creative Commons. Para ver una copia de esta licencia, visite http://creativecommons.org/ licenses/by-nc/3 